\title{
Analysis of the Effective Dynamic Properties of Particulate Composites with Respect to Constituent Properties
}

\author{
Mohammad Rahimzadeh ${ }^{\text {a* }}$ (D) \\ a Department of Mechanical Engineering, Faculty of Engineering, Golestan University, Gorgan, Iran. Email: m.rahimzadeh@gu.ac.ir \\ * Corresponding author
}

https://doi.org/10.1590/1679-78256893

\begin{abstract}
The propagation of longitudinal and shear elastic waves through a multi-phase material was studied and the effective elastic properties of the medium were evaluated. The distribution of the reinforcing inclusions was considered random throughout the matrix. The effective dynamic properties of the composites, including their effective bulk and shear moduli and effective densities, were examined along with the effective phase velocity and attenuation of the incident $P$ and $S$ waves. The Sabina-Willis model was employed to study the wave propagation behavior, and the model performance was analyzed through comparison with experimental data from the literature. The results indicated that wave propagation significantly depended on the physical and mechanical properties of inclusions relative to those of the matrix and the normalized wave number of the propagated elastic wave. Moreover, despite the fact that the elastic properties of the incidence in the $\mathrm{P}$ and $\mathrm{S}$ waves exhibited a similar trend, their values differed significantly. The results can serve as a design criterion for composite materials under dynamic loading.
\end{abstract}

\section{Keywords}

Particulate composites, Effective dynamic properties, Random media, P \& S waves.

\section{Graphical Abstract}

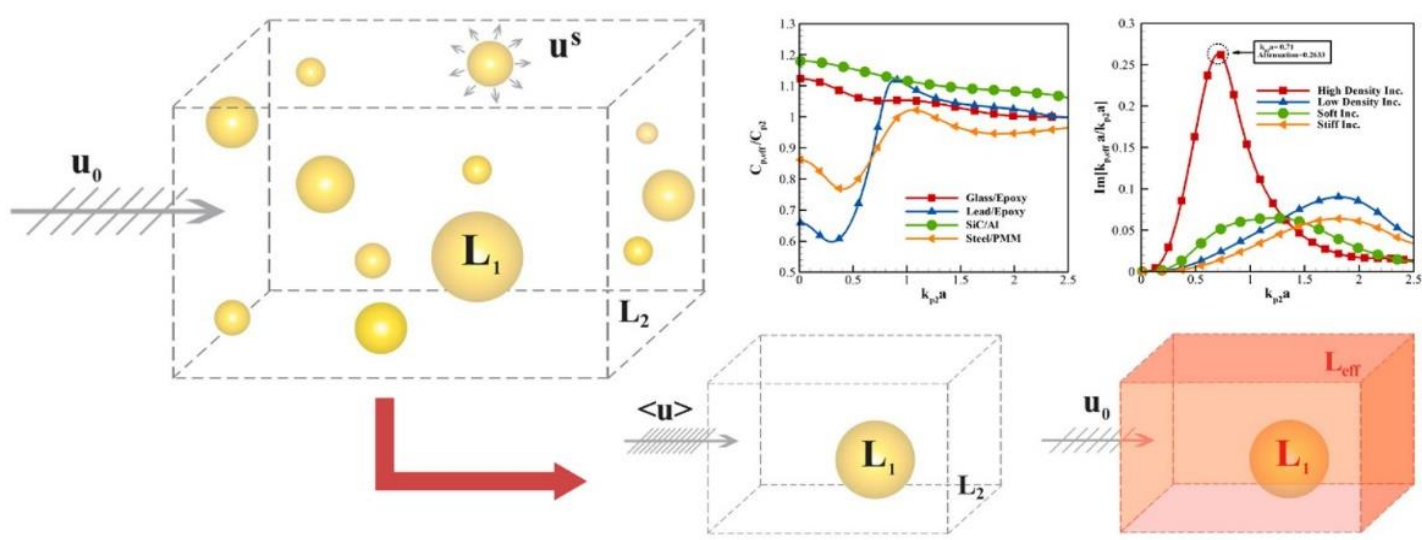




\section{INTRODUCTION}

Having a wide range of industrial applications, composite materials have become quite prevalent owing to their substantial lightweight and the fact that their mechanical properties can be tailored to intended loading situations. Despite the unique advantages of fiber composites, their higher costs have made other composites such as particulate composites more attractive for research. Particulate composites are widely used in aerospace structures, including commercial and military aircrafts and spaceships. The importance of understanding the dynamic behavior of such composites becomes evident by considering the dynamic nature of loading conditions in most of the mentioned applications. Furthermore, understanding the elastic wave propagation behavior in such media helps with nondestructive fault and crack detection substantially.

Wave propagation through inhomogeneous media leads to scattering, which causes the velocity and attenuation of the mean wave to be dependent on the frequency of the propagated wave. Foldy (1945) and Lax (1951), estimated the phase velocity and attenuation of a coherent wave in terms of filler concentration and scattering of the wave from a particle along the propagation direction. Their results were later improved by Waterman and Truell (1961). They estimated the multiple scattering behavior by using the scattering amplitude in a backward direction and the scattering amplitude along the propagation direction.

The resulting model was extended by Lloyd and Berry (1967), who showed that the equations derived by Waterman and Truell contained an integration error. They adopted an energy density approach to derive the wave number in an inhomogeneous medium. Their final equation included one additional integral term compared to Waterman and Truell's model. The results were, to some extent, more reliable for higher volume fractions.

Additionally, various studies based on the above models were focused on the interface effects. Aiming to analyze such effects, Datta et al. (1988) used Foldy's theory to analyze longitudinal and shear plane-wave propagation in composites containing randomly distributed spherical inclusions. Sato and Shindo (2003) used Lax's approximation (QCA) to analyze the scattering of harmonic waves in a metal-matrix composite considering graded interfaces. Wei and Huang (2004), revealed that the viscoelastic interface had a noticeable effect on the wave number and the elastic moduli of the composites. They also showed that, while attenuation effects dominated at low frequencies, the multiple scattering effects dominated at high frequencies. These approaches to modeling the multiple scattering phenomenon offer acceptable accuracy at low volume fractions of inclusions and low propagation frequencies. Nevertheless, they are unable to provide a satisfactory estimate of the final static properties of the inhomogeneous medium in the frequency ranges close to zero. Therefore, one cannot ensure the validity of the results obtained from these approaches when low frequencies are involved.

Following Waterman and Truell's work, Bringi et al. (1982) and Varadan et al. (1985) developed the T-matrix approach to study multiple scattering using the QCA and a pair correlation function. Mal and Bose (1974) analyzed the propagation properties of the mean wave using a statistical method. They also analyzed the effects of inclusion's slip on the average parameters of the inhomogeneous media. Determining a correlation function between two particles in the multiple scattering phenomenon has been the focus of many research works, examples of which are Markov and Willis (1998), Markov (1999), and Liu and Turner (2008).

Willis (1980) presented the relations for solving elastodynamic problems of wave scattering through inhomogeneous media in terms of integral equations. Among the difficulties associated with the implementation of the above methods is the determination of a pair correlation function for high concentration cases. The effective elastodynamic constants of such media are entirely dependent on this function determination. However, such methods are capable of determining the final dynamic properties of inhomogeneous media at low frequencies and volume fractions. Berryman (1980) analyzed the wave propagation through a multi-phase medium to determine its effective static properties. His approach was to set the scattering wave field equal to zero to determine the final elastic constants. He showed that the results obtained from this approach were more accurate compared with those obtained from Kuster and Toksöz's (1974) method. The model outputs were compared with other methods, such as those reported by Hill (1965) and Budiansky (1965).

Sabina and Willis (1988) reported the first classic self-consistent analysis using an effective medium approach, which yielded acceptable results at high filler concentrations. In their approach, the mean wave properties were gained through an iterative method. They showed that the resulting self-consistent equations, yielded acceptable results when the elastic wavelength was at least four times the radius of the inclusions in the inhomogeneous medium. Based on a self-consistent approach, Kim et al. (1995) presented an approach analogous to the approximation in alloy physics. Compared with the Waterman and Truell model, the analytical results reported by Kim et al. were more consistent with Kinra's experimental results. These methods presented zero-frequency elastic properties similar to those obtained from Hill and Budiansky's static analysis. 
Various micromechanics methods such as generalized self-consistent, and Mori-Tanaka methods exist for the approximation of elastostatic moduli of composites. Christensen (1990) showed that the generalized self-consistent method is more accurate even at high filler concentrations. Yang (2003) further extended this method to study the planewave multiple scattering through random media. He showed that, for dense composites, the model performed better than the mean-field methods. In addition, Kanaun and Levin (2007) used the effective field and medium approaches to derive the equations describing elastic wave scattering through such composites. Rahimzadeh and Daneshjoo (2014) conducted a comparative study on four different theoretical models for the prediction of propagation behavior of the elastic P-wave in composites reinforced by randomly distributed inclusions. Luppé et al. (2017) studied the effects of the correlation among scatterers on the propagation of the average wave through a viscoelastic medium containing spherical or cylindrical inclusions.

Various experimental studies have also been published on the plane propagation of elastic waves through particulate composites. Kinra et al. $(1982,1998)$ conducted some experimental studies to analyze the ultrasonic wave propagation in composites with inclusions randomly distributed through the matrix. Layman et al. (2006) experimentally measured the wave properties in such composites. Through a comparison, they showed that the dynamic generalized self-consistent model was more accurate for higher volume fractions. Using experimental data, Li et al. (2017) showed that particle interactions played a major role in the propagation behavior of ultrasonic waves and their attenuation, which heavily affects the viscoelastic properties of particulate composites. Other research works considered different particle geometries. Mykhas'kiv et al. $(2010,2018)$ considered composites containing penny-shaped inclusions and analyzed variations in the wave properties with respect to the normalized wave number. Using a Foldy-type relation and the boundary-element method for a single inclusion, they proposed an approach to analyzing the effective dynamic parameters of the two-phase media. Both random and parallel aligned inclusion distributions were considered.

The present study analyzed the effective dynamic properties of particulate composites containing inclusions softer, stiffer, lighter, and heavier than the matrix. The analysis was carried out for the two different incidences of the P or S waves, and the effective elastic properties were examined for the two cases. The effective phase velocity and effective attenuation were examined at a moderate volume fraction of $c=30 \%$. Next, the effective properties were analyzed based on the normalized wave number of $k_{p 2} a=2$ for various volume fractions. The Sabina-Willis model results were also compared against published experimental data.

\section{Solution Approaches to Multiple Scattering Problems}

Since the exact solutions are impossible in the case of media containing randomly distributed inhomogeneity, approximate methods have become the primary approach to solving these problems (Rahimzadeh, 2013). Among other approximate methods, self-consistent methods are more widely used owing to their remarkable benefits. These methods are based on some simplifying assumptions, which transform a multiple scattering problem and the mutual effects of inhomogeneities into a simple issue of scattering from a single inhomogeneity. The self-consistent methods are superior in that they are able to convert a complex multi-particle problem to a simple problem with only one particle. The assumptions used by these methods were examined and improved by many researchers so that the model's prediction of the final behavior of composite materials can be enhanced and validated against experimental data. These methods can be classified into two main groups, namely the effective field and medium methods.

\subsection{Effective Field Methods}

These methods use the following assumptions:

- Each inclusion behaves similarly to an isolated inclusion inside the matrix.

- The effects of other inclusions are taken into account by considering an effective field applied to the isolated inclusion (Kanaun and Levin, 2005).

The second assumption forms the basis of many research works on the topic. The most straightforward approach, known as the QCA, is to consider the effective field as a planar wave field that is uniformly exerted on all of the inclusions. Contrary to the effective medium methods, the effective field methods depend on how inclusions are distributed through the medium. The reason is because these methods employ a correlation function to estimate the effective field.

\subsection{Effective Medium Methods}

Effective medium methods rely on the following two simplifying assumptions (Kanaun et al., 2004): 
- $\quad$ Each particle in the inhomogeneous medium behaves similarly to separate inclusion inside a homogeneous medium whose properties are similar to those of the inhomogeneous medium.

- $\quad$ The average wave field in the inhomogeneous medium is consistent with the wave field propagated through the homogeneous medium (self-consistency condition).

The first assumption implies that, material properties in an inclusion surrounding are replaced by a homogeneous medium whose properties are equivalent to the effective properties of the entire inhomogeneous medium. In other words, if the elastic property tensor and the density of the matrix are represented by $\mathbf{L}_{2}$ and $\rho_{2}$, respectively, and the properties of the spherical inhomogeneities inside this medium is represented by $\mathbf{L}_{1}$ and $\rho_{1}$, then it is assumed that any inclusion inside the medium is placed in a homogeneous medium having the final properties of the entire composite material, i.e., $\mathbf{L}_{\text {eff }}$ and $\rho_{\text {eff }}$. The second assumption used by this family of methods has other versions too. These methods are shown in Figure 1.

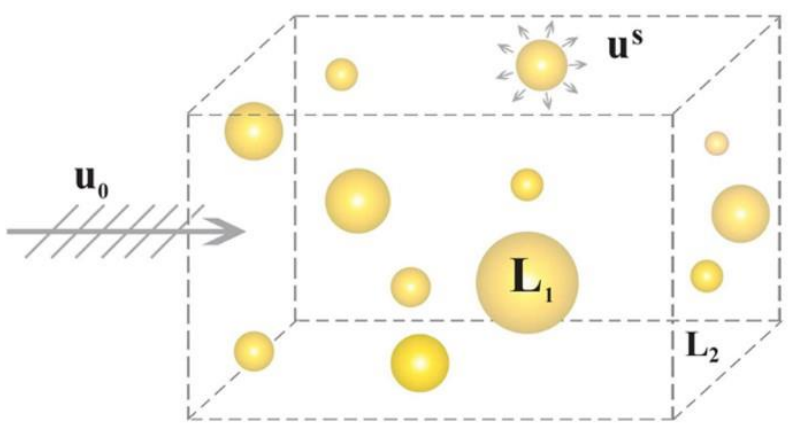

(a)

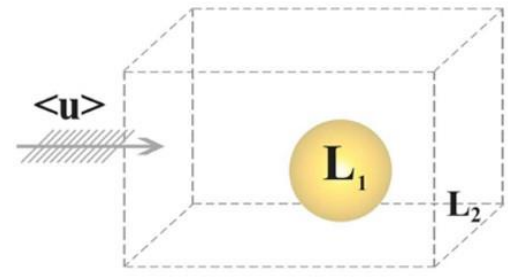

(c)

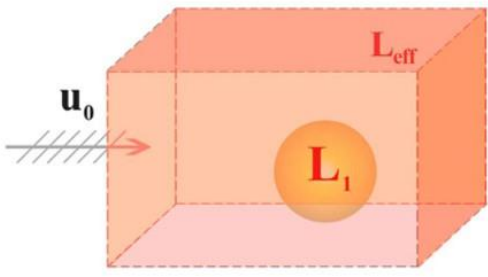

(b)

Figure 1: Schematics of a) elastic wave propagation through the composite material containing scatterers, b) effective medium schematic model, and c) effective field schematic model.

\section{The Sabina-Willis Model}

Consider an inhomogeneous composite material consisting of a matrix and a random distribution of $n$ different kinds of other particles. In other words, the material is composed of $n+1$ different phases. The density and elastic moduli tensor of the matrix are represented by $r_{n+1}$ and $\mathbf{L}_{n+1}$, respectively, and the density and elastic modulus tensor of each inclusion of type $r$ are represented by $\rho_{r}$ and $\mathbf{L}_{r}$ with $r=1,2, \ldots, n$. It is assumed that inclusions of type $r$ are the same shape and size. In addition, each inclusion of kind $r$ occupies the region $\mathbf{x}^{\prime}+\Omega_{r}$ of space, where $\mathbf{x}^{\prime}$ is the inclusion center, which is considered a random variable, and $\Omega_{r}$ represents the shape and size of the inclusion.

The probability density function of inclusions of type $r$ is denoted by $n_{r}$, which is independent of the random variable $\mathrm{x}^{\prime}$ This function defines the probability of finding the center of any inclusion of kind $r$ at $\mathrm{x}^{\prime}$. Additionally, the probability of any point in space being in a medium of kind $r$ is $c_{r}=n_{r}+\Omega_{r}$, which is the concentration of inclusions of type $r$. The volume fraction $c_{n+1}$ of the matrix is: 
$\sum_{r=1}^{n+1} c_{r}=1$

For a determined load, the displacement field $\mathbf{u}$ of a multi-phase composite depends on how the inclusions are distributed. Therefore, given the random distribution of inclusions in the problem, the mean displacement field $\langle\mathbf{u}\rangle$ was deemed instead of exact displacements. The response of the medium to an arbitrary loading satisfies the equation of motion, which, when body forces are neglected, is given by:

$\nabla \cdot \sigma=\dot{\mathbf{p}}$

According to linear elasticity and the momentum-velocity relations:

$\boldsymbol{\sigma}=\mathbf{L e}$

Where $\rho$ is the density and $\dot{\mathbf{u}}$ is the velocity. The elastic modulus tensor $\mathbf{L}$ and $\rho$, which are functions of $\mathbf{x}$, are given by:

$\mathbf{P}=\rho \dot{\mathbf{u}}$

$\mathbf{L}(\mathbf{x})=\sum_{r=1}^{n+1} \mathbf{L}_{n} f_{r}(\mathbf{x})$

$\rho(\mathbf{x})=\sum_{r=1}^{n+1} \rho_{n} f_{r}(\mathbf{x})$

Where:

$f_{r}(\mathbf{x})=\left\{\begin{array}{rr}1 ; & \mathbf{x} \in \text { material } r \\ 0 ; & \text { otherwise }\end{array}\right.$

The equation governing the average response of the composite is obtained by averaging Eq. (2) as follows:

$\nabla \cdot\langle\boldsymbol{\sigma}\rangle=\langle\dot{\mathbf{p}}\rangle$

Accordingly, $\langle\mathbf{u}\rangle$ can be determined if effective constitutive relations similar to Eqs. (3) and (4) can be established between $\boldsymbol{\sigma}$ and $\langle\dot{\mathbf{p}}\rangle$ on the one hand, and $\langle\mathbf{e}\rangle$ and $\langle\dot{\mathbf{u}}\rangle$ on the other. If the total average of $\mathbf{e}$ at the point $\mathbf{x}$ in the studied medium is denoted by $\left\langle\mathbf{e}_{r}\right\rangle$, and $\mathbf{x}$ is located in a phase of type $r$, then:

$\langle\mathbf{e}\rangle=\sum_{r=1}^{n+1} \mathrm{c}_{r}\langle\mathbf{e}\rangle_{r}$

In a similar fashion, the following relations are obtained for the other parameters:

$\langle\dot{\mathbf{u}}\rangle=\sum_{r=1}^{n+1} \mathrm{c}_{r}\langle\dot{\mathbf{u}}\rangle_{r}$ 


$$
\begin{aligned}
& \langle\boldsymbol{\sigma}\rangle=\sum_{r=1}^{n+1} \mathrm{c}_{r}\langle\boldsymbol{\sigma}\rangle_{r}=\sum_{r=1}^{n+1} \mathbf{L}_{r}\langle\mathbf{e}\rangle_{r} \\
& \langle\mathbf{p}\rangle=\sum_{r=1}^{n+1} \mathrm{c}_{r}\langle\mathbf{p}\rangle_{r}=\sum_{r=1}^{n+1} c_{r} \rho_{r}\langle\dot{\mathbf{u}}\rangle_{r}
\end{aligned}
$$

Similar to what Hill did in his static analysis, eliminating $c_{n+1}\left\langle\mathbf{e}_{n+1}\right\rangle$ from Eqs. (9) and (11), and $c_{n+1}\left\langle\dot{\mathbf{u}}_{n+1}\right\rangle$ from Euations (10) and (12) leads to:

$$
\begin{aligned}
& \langle\boldsymbol{\sigma}\rangle=\mathbf{L}_{n+1}\langle\mathbf{e}\rangle+\sum_{r=1}^{n} c_{r}\left(\mathbf{L}_{r}-\mathbf{L}_{n+1}\right)\langle\mathbf{e}\rangle_{r} \\
& \langle\mathbf{p}\rangle=\rho_{n+1}\langle\dot{\mathbf{u}}\rangle+\sum_{r=1}^{n} c_{r}\left(\rho_{r}-\rho_{n+1}\right)\langle\dot{\mathbf{u}}\rangle_{r}
\end{aligned}
$$

Therefore, the fundamental relations of the problem can be derived if $\left\langle\mathbf{e}_{r}\right\rangle$ and $\left\langle\dot{\mathbf{u}}_{r}\right\rangle$ can be written in terms of $\langle\mathbf{e}\rangle$ and $\langle\dot{\mathbf{u}}\rangle$. A common method to tackling this problem is to deem an isolated inclusion inside a homogeneous medium with the same properties as the composite material. Based on the above equations, the effective elastic properties of the multi-phase material can be obtained as below:

$$
\begin{aligned}
& \langle\mathbf{p}\rangle=\rho_{n+1}\langle\dot{\mathbf{u}}\rangle+\sum_{r=1}^{n} c_{r}\left(\rho_{r}-\rho_{n+1}\right)\langle\dot{\mathbf{u}}\rangle_{r} \\
& \rho_{\text {eff }}=\rho_{n+1}+\sum_{r=1}^{n+1} c_{r} h_{r}(g) h_{r}(-g)\left(\rho_{r}-\rho_{n+1}\right)^{-1}\left[\mathbf{I}+\mathbf{M}_{x}^{r}\left(\rho-\rho_{\text {eff }}\right)\right]
\end{aligned}
$$

The general equations above can be rewritten for the special case of identical inclusions with radius $a$ randomly distributed throughout the matrix. Denoting the effective shear modulus and bulk modulus of the matrix by $\kappa_{2}$ and $\mu_{2}$, respectively, and those of the inclusions by $\kappa_{1}$ and $\mu_{1}$, respectively, one obtains the following relations based on the general equations of the Sabina-Willis model:

$$
\begin{aligned}
& \kappa_{\text {eff }}=\kappa_{2}+\frac{c_{1} h_{1}(g) h_{1}(-g)\left(\kappa_{1}-\kappa_{2}\right)}{1+3\left(\kappa_{1}-\kappa_{e f f}\right) \varepsilon_{a} /\left(3 \kappa_{\text {eff }}+4 \mu_{\text {eff }}\right)} \\
& \mu_{\text {eff }}=\frac{\mu_{2}+c_{1} h_{1}(g) h_{1}(-g)\left(\mu_{1}-\mu_{2}\right)}{1+2\left(\mu_{1}-\mu_{e f f}\right)\left[2 \mu_{e f f} \varepsilon_{\alpha}+\left(3 \kappa_{e f f}+4 \mu_{e f f}\right) \varepsilon_{\beta}\right] / 5 \mu_{e f f}\left(3 \kappa_{e f f}+4 \mu_{\text {eff }}\right)} \\
& \rho_{\text {eff }}=\rho_{2}+\frac{c_{1} h_{1}(g) h_{1}(-g)\left(\rho_{1}-\rho_{2}\right)}{1+\left(\rho_{1}-\rho_{\text {eff }}\right)\left[3-\varepsilon_{\alpha}-2 \varepsilon_{\beta}\right] / 3 \rho_{\text {eff }}}
\end{aligned}
$$


The function $h_{1}(g)$ for a spherical inclusion is defined as:

$h_{1}(g)=3(\sin g a-g a \cos g a) /(g a)^{3}$

where $g$ is replaced by the longitudinal wave number $k$ if there is a longitudinal incident wave and by the shear wave number $K$ if there is a shear incident wave. In addition, from the above equations:

$\varepsilon_{\alpha}=\frac{3\left(1-i k_{e f f} a\right)\left[\sin \left(k_{e f f} a\right)-k_{e f f} a \cos \left(k_{e f f} a\right)\right] e^{i k_{e f f} a}}{\left(k_{e f f} a\right)^{3}}$

$\varepsilon_{\beta}=\frac{3\left(1-i K_{e f f} a\right)\left[\sin \left(K_{e f f} a\right)-K_{e f f} a \cos \left(K_{e f f} a\right)\right] e^{i K_{\text {eff }} a}}{\left(K_{\text {eff }} a\right)^{3}}$

The longitudinal and shear wave numbers of the effective medium based on the obtained elastic properties can be expressed as:

$k_{e f f}=\omega\left[\left(3 \kappa_{e f f}+4 \mu_{e f f}\right) / 3 \rho_{e f f}\right]^{-1 / 2}$

$K_{\text {eff }}=\omega\left[\mu_{\text {eff }} / \rho_{\text {eff }}\right]^{-1 / 2}$

These equations can be solved through iteration. Numerical methods for nonlinear equations, such as the GuassNewton method, can also be used for this purpose.

\section{Results and Discussion}

Equations (17)- (20) were solved through iteration. First, the model was solved for the composite consisting of steel spherical inclusions and a polymetilmetacrelate (PMM) matrix. The filler concentration was $c=15.2 \%$ The physical and mechanical properties of each constituent part of the composite are listed in Table 1. Figure 2 shows the normalized phase velocity results with respect to the normalized wave number and compares them with the experimental data reported by Kinra (1985). It is observed that the model provided a better prediction of wave propagation behavior at lower normalized wave numbers. Based on the assumptions of the Sabina-Willis model, the results are valid up to $k a=\pi / 2$. Since $k a=2 \pi / \delta$, where $\delta=\lambda / a$, the maximum wavelength can be up to two times of the inclusions diameter. In other words, the Sabina-Willis model can be employed for long and moderate wavelengths; the modeling error grows at shorter wavelengths. To investigate the effective dynamic properties, different combinations of reinforcing inclusions and matrix were considered (Table 1). Four composites of Glass/Epoxy, Lead/Epoxy, SiC/Al, and Steel/PMM were considered at $c=30 \%$ volume fractions. Their normalized phase velocity and attenuation as functions of the normalized wave number are shown in figure 3 . The phase velocity curve is observed to exhibit a higher sensitivity to variations in the normalized wave number for Steel/PMM. This composite also experienced the highest attenuation value of 0.2274 , which occurred at $k_{p 2} a=0.63$ Understanding the effects of the constituents of a composite is necessary for the analysis of their behavior during the propagation of shear and longitudinal waves. 
Table 1 Physical and mechanical properties of the constituents of the studied composite materials.

\begin{tabular}{|c|c|c|c|c|c|c|c|}
\hline \multirow[b]{2}{*}{ Case } & \multirow[b]{2}{*}{ Volume fraction } & \multicolumn{3}{|c|}{ Inclusions Properties } & \multicolumn{3}{|c|}{ Matrix Properties } \\
\hline & & $\begin{array}{c}\kappa_{1} \\
(\mathrm{GPa})\end{array}$ & $\begin{array}{c}\mu_{1} \\
\text { (GPa) }\end{array}$ & $\begin{array}{c}\rho_{1} \\
\left(\mathrm{Kg} / \mathrm{m}^{3}\right)\end{array}$ & $\begin{array}{c}\kappa_{2} \\
(\mathrm{GPa})\end{array}$ & $\begin{array}{c}\mu_{2} \\
\text { (GPa) }\end{array}$ & $\begin{array}{c}\rho_{2} \\
\left(\mathrm{Kg} / \mathrm{m}^{3}\right)\end{array}$ \\
\hline Steel/PMM & $15.2 \%, 30 \%$ & 167.38 & 80.87 & 7800 & 5.33 & 2.02 & 1160 \\
\hline Lead/Epoxy & $30 \%$ & 44.05 & 8.35 & 11300 & 6.07 & 1.73 & 1202 \\
\hline $\mathrm{SiC} / \mathrm{Al}$ & $30 \%$ & 223.40 & 188.10 & 3181 & 75.17 & 26.50 & 2706 \\
\hline Glass/Epoxy & $30 \%$ & 34.59 & 26.16 & 2492 & 5.50 & 1.59 & 1180 \\
\hline Soft Inc. & $30 \%$ & $\kappa_{2} / 4$ & $\mu_{2} / 4$ & $\rho_{2}$ & 75.20 & 26.50 & 2706 \\
\hline Stiff Inc. & $30 \%$ & $4 \kappa_{2}$ & $4 \mu_{2}$ & $\rho_{2}$ & 75.20 & 26.50 & 2706 \\
\hline Low Density Inc. & $30 \%$ & $\kappa_{2}$ & $\mu_{2}$ & $\rho_{2} / 4$ & 75.20 & 26.50 & 2706 \\
\hline High Density Inc. & $30 \%$ & $\kappa_{2}$ & $\mu_{2}$ & $4 \rho_{2}$ & 75.20 & 26.50 & 2706 \\
\hline
\end{tabular}

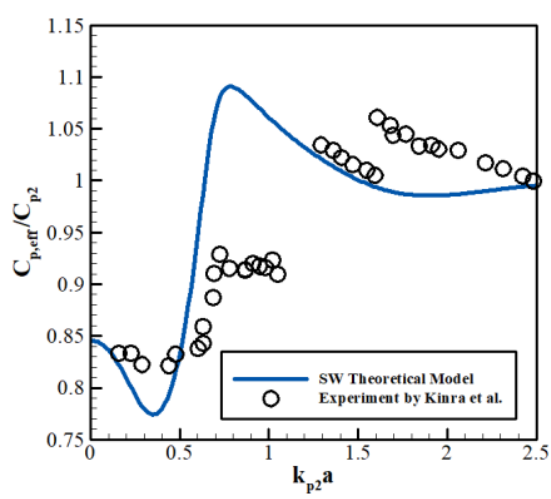

(a)

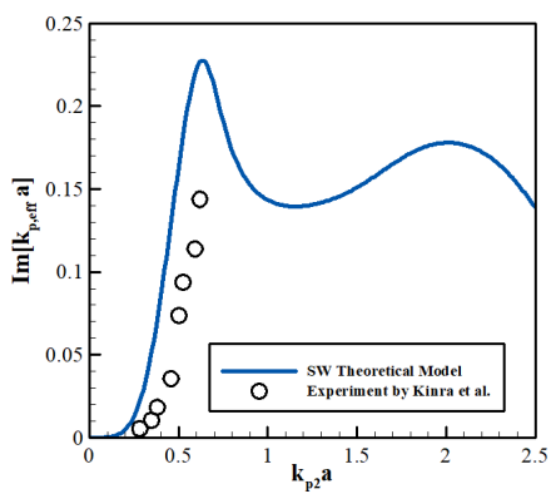

(b)

Figure 2: Comparison of the (a) normalized phase velocity and (b) attenuation results as a function of the normalized wave number obtained from the analytical Sabina-Willis model with Kinra et al. experimental data for the Steel/PMM composite.

Accordingly, these parameters were studied in four different cases in what follows. First, the elastic properties of inclusions, such as their bulk and shear moduli, were considered lower than those of the matrix (soft inclusions). The next case considered them to be higher than the elastic properties of the matrix (stiff inclusions). In the third case, the elastic properties of both the inclusions and the matrix were considered the same, but the density of inclusions was lower and higher than that of the matrix (light and heavy inclusions). For the analysis of the normalized propagated wave number, the volume fraction of inclusions was selected to be $c=30 \%$. All of the parameters of the average wave were normalized with respect to the matrix medium. Similarly, the frequency axis in all of the graphs was considered as the normalized longitudinal wave number.

Figure 4 shows the variations in the dimensionless velocity of the longitudinal and shear waves with respect to the normalized wave number for the composites containing the above mentioned inclusions. It is observed from the figure that in all of the cases, when the propagated wave frequency was in the low-frequency or long-wavelength range, the phase velocity variations were negligible compared to the frequency variation. In fact, when exposed to long wavelengths, the composite exhibited static behavior. This can be justified by considering the decrease in scattering in the case of long wavelengths. As the propagated wave frequency reached the moderate-frequency or moderate-wavelength range, especially in the case with high density inclusions, the phase velocity became highly sensitive to the normalized wave number. Multiple scattering was dominated owing to the wavelength of the propagated wave. Therefore, this case experienced the largest variations in the composite phase velocity with frequency. The composite with stiff inclusions had the highest normalized phase velocity, which was equal to 1.198 and 1.228 for the incident $P$ and $S$ waves respectively. As the frequency increased and reached the moderate-frequency range, the normalized phase velocity curve of the composites containing inclusions heavier and lighter than the matrix assumed decreasing and increasing slopes, respectively. In all cases, as the normalized wave number increased (and the wavelength of the propagated wave became smaller compared to the inclusion dimensions), the normalized phase velocity approached unity. 
Figure 5 shows the normalized longitudinal and shear wave attenuation as a function of the normalized wave number. Starting from zero, the curve followed an increasing trend with frequency until it reached a maximum, after which it exhibited a downward slope as the frequency increased. With heavy inclusions, the normalized attenuation for the incident $\mathrm{P}$ and $\mathrm{S}$ waves, peaked at 0.2633 for $k_{p 2} a=0.71$ and 0.1849 for $k_{s 2} a=1.3$ respectively. The normalized attenuation for the cases with soft and stiff inclusions was remarkably lower than for the other cases. With soft inclusions, the normalized attenuation for the incident $\mathrm{P}$ and $\mathrm{S}$ waves peaked at 0.0649 for $k_{p 2} a=1.23$ and 0.0397 for $k_{s 2} a=1.37$ respectively.

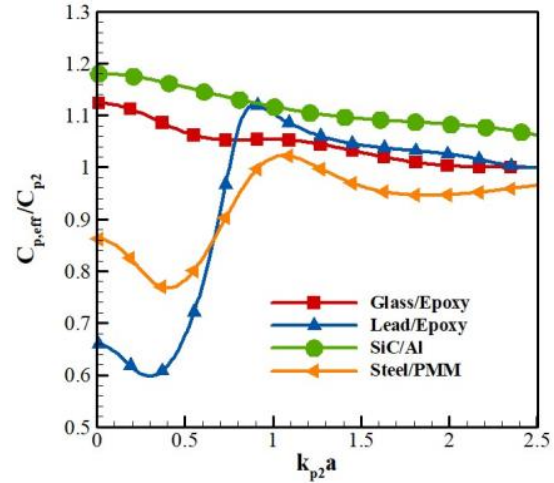

(a)

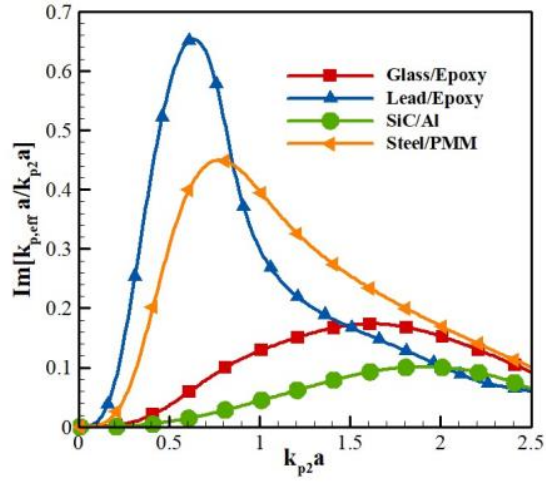

(b)

Figure 3: Variations in the (a) normalized phase velocity and (b) attenuation of Glass/Epoxy, Lead/Epoxy, SiC/Al and Steel/PMM composites as a function of the normalized wave number.

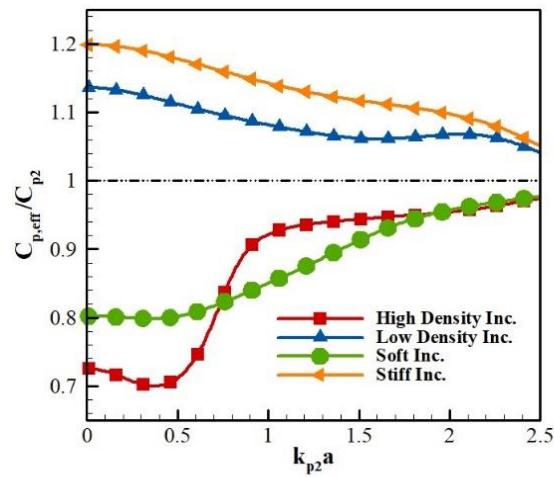

(a)

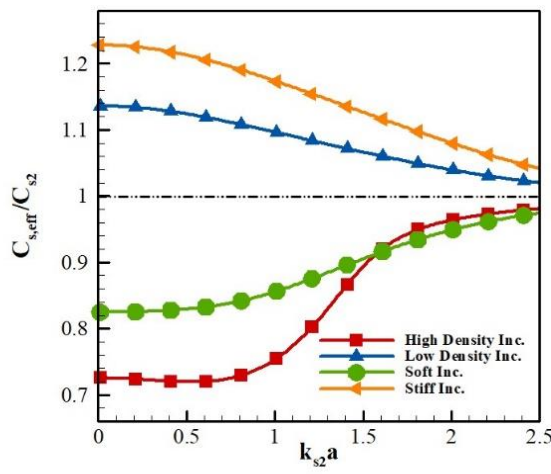

(b)

Figure 4: Variations in the normalized phase velocity as a function of the normalized longitudinal wave number for the cases with soft, stiff, light, and heavy inclusions and $30 \%$ volume fraction. (a) P wave incidence. (b) S wave incidence.

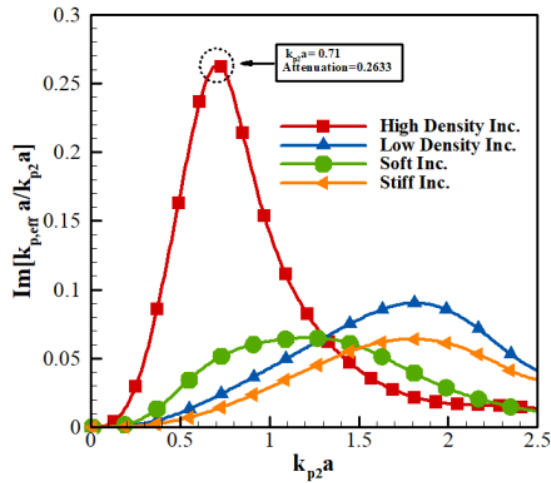

(a)

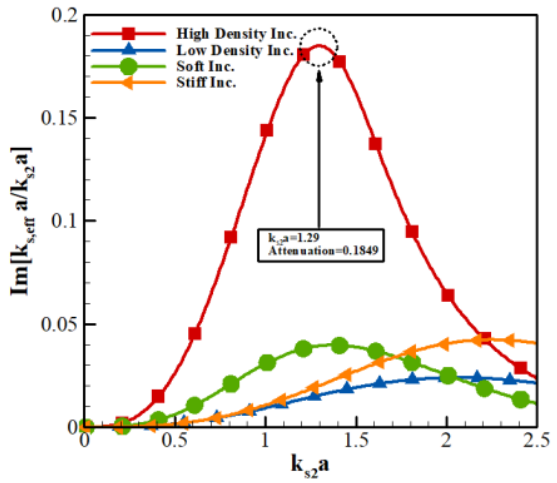

(b)

Figure 5: Variations in the normalized attenuation as a function of the normalized longitudinal wave number for the cases with soft, stiff, light, and heavy inclusions and $30 \%$ volume fraction. (a) P wave incidence. (b) S wave incidence. 
For the cases containing soft and stiff inclusions, the attenuation peaks can be attributed to the elastic resonance of inclusions inside the medium. Given the fact that the constituent phases were of equal density, this resonant mode cannot be considered the rigid-body resonant mode of the inclusions. Moreover, based on the Kramers-Kronig relations, variations in the attenuation curve changed the phase velocity curve. From the fact that the elastic properties of the two constituent parts were the same and that there was a significant density difference between the light and heavy inclusions, the curve peak can be concluded to belong to the rigid-body resonance of the spherical inclusion inside the medium. The lowest resonant frequency is observed in the curve of the composite that contained heavy inclusions.

Figure 6 shows variations in the normalized bulk modulus of the effective medium as a function of the normalized wave number for $c=30 \%$ volume fraction. The variations in the bulk modulus of the material were insignificant in the lowfrequency range. As the incident wave frequency increased, the values grew larger, whereas the corresponding variations in the composite containing stiff inclusions exhibited a decreasing trend. It is observed that as the frequency of the propagated wave increased or the reinforcing inclusions became smaller, the effective bulk modulus approached the bulk modulus of the matrix. At low frequencies, the effective bulk modulus was almost the same as the static bulk modulus of the composite material, which was equal to 0.6264 and 1.4037 in the cases that contained soft and stiff inclusions, respectively.

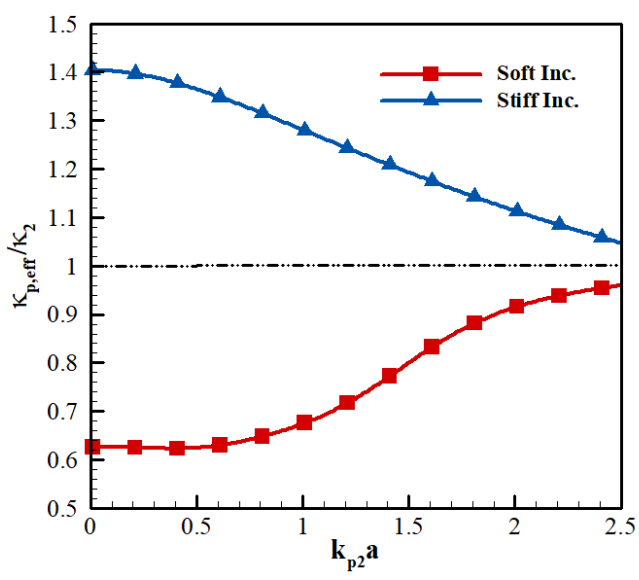

(a)

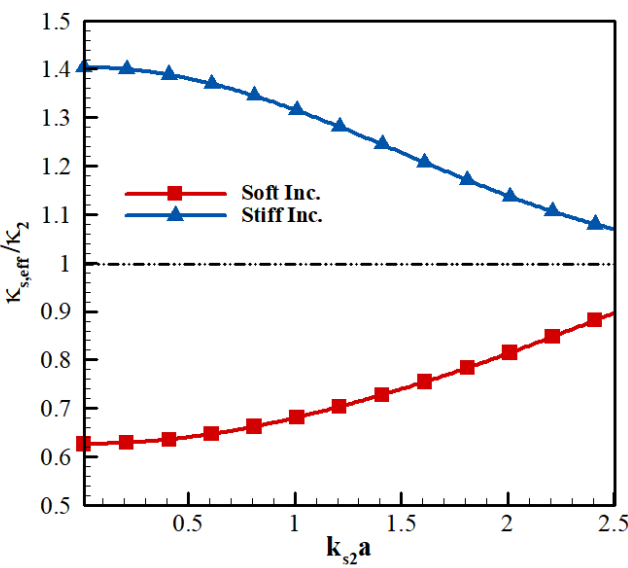

(b)

Figure 6: Dependence of the normalized effective bulk modulus on the normalized wave number for the cases with soft and stiff inclusions and $30 \%$ volume fraction. (a) $\mathrm{P}$ wave incidence. (b) S wave incidence.

Variations in the normalized shear modulus are shown in Figure 7. As expected, the effective shear modulus was reduced by adding soft inclusions and increased by stiff inclusions; the resulting values were 0.815 and 1.23 , respectively, in the static case (zero frequency). In addition, in the case of P incident wave, the effective shear modulus curve appeared to be more sensitive to the wave frequency.

Figure 8 shows the dimensionless effective density as a function of the normalized wave number for the cases containing light and heavy inclusions at 30\% volume fraction. In the static case, the effective densities of the composites containing light and heavy inclusions were 0.7750 and 1.9002, respectively. It is observed that changes in the low frequency range led to an insignificant change in the effective density for both cases. For the case with heavy inclusions, the changes in moderate wavelengths are observed to be more pronounced; from $k_{p 2} a=0.3$ to 1 , the effective density dropped by $43 \%$ in the case of the $P$ wave.

Figure 9 shows variations in the dimensionless velocity and attenuation as a function of inclusions concentration for $k_{p 2} a=2$. It is observed that, as the concentration of stiff and low-density inclusions increased, the normalized phase velocity exhibited an upward slope and it had a downward slope for higher volume fractions of the soft and high-density inclusions. This is explained by the fact that the effective wave number of the medium is directly proportional to the density of the effective medium squared and inversely proportional to the elastic constants of the effective medium. 


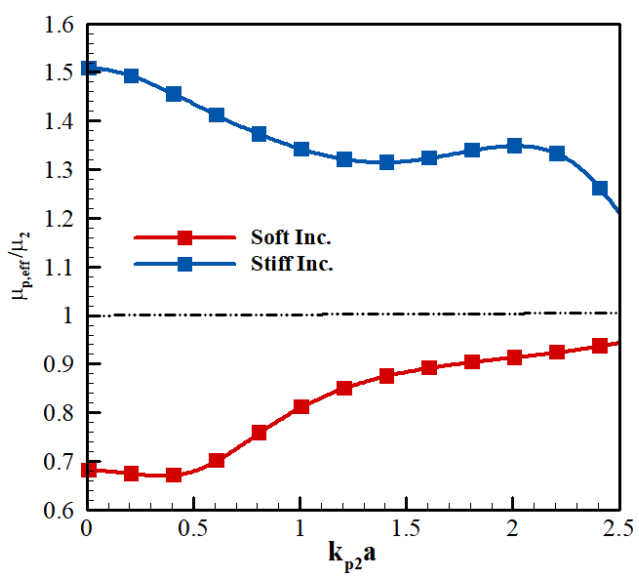

(a)

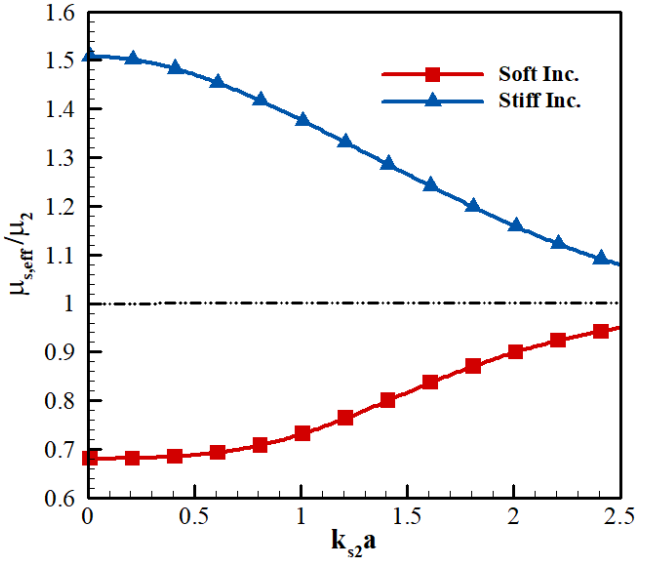

(b)

Figure 7: Dependence of the normalized effective shear modulus on the normalized wave number for the cases with soft and stiff inclusions and $30 \%$ volume fraction. (a) $\mathrm{P}$ wave incidence. (b) $\mathrm{S}$ wave incidence.

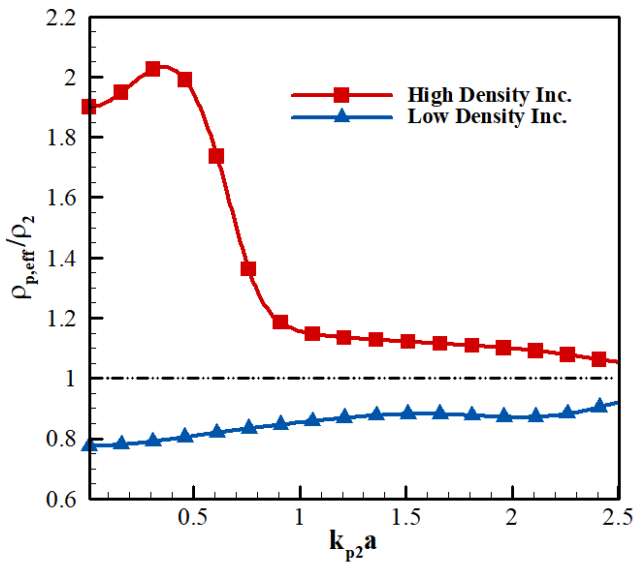

(a)

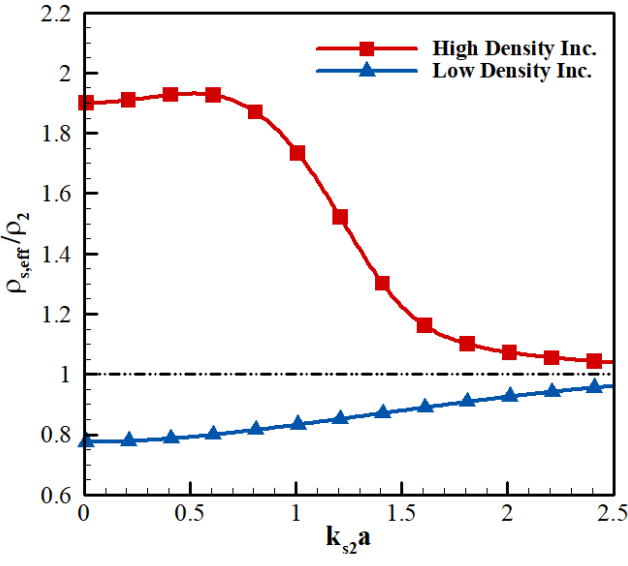

(b)

Figure 8: Dependence of the normalized effective density on the normalized wave number for the cases with light and heavy inclusions and $30 \%$ volume fraction. (a) $\mathrm{P}$ wave incidence. (b) S wave incidence.

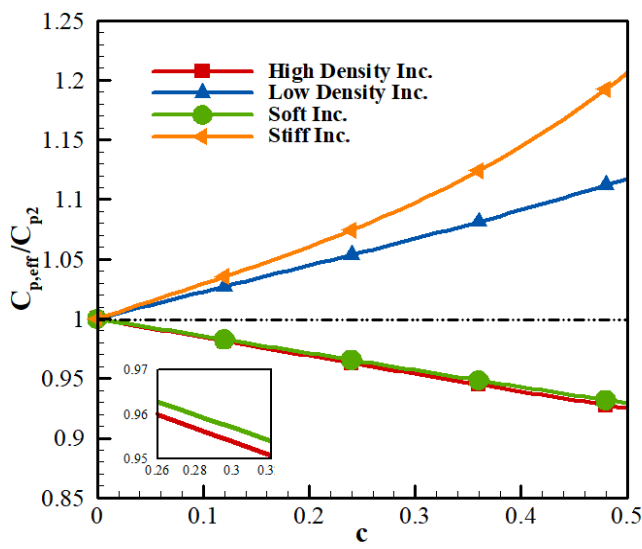

(a)

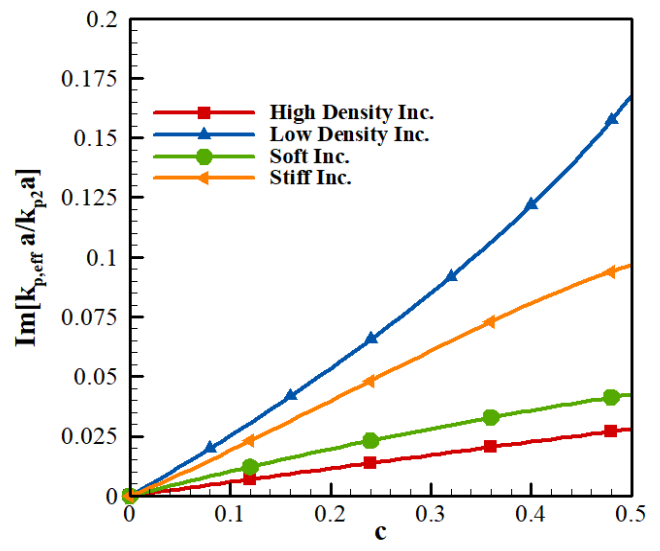

(b)

Figure 9: Variations in the(a) normalized phase velocity and (b) attenuation as a function of the volume fraction of composite material for the cases with soft, stiff, light, and heavy inclusions and the wave number $k_{p 2} a=2$.

At a zero volume fraction, for which the composite material can be considered as the matrix, the normalized phase velocity was correctly equal to one. In addition, at low volume fractions, the curves of the normalized phase velocity of the light and stiff inclusions and that of the heavy and soft inclusions exhibited only a small difference. It is observed that the highest attenuation occurred for the case containing light inclusions. Starting from zero value, the normalized attenuation 
variation curve followed a mostly increasing trend. The lowest attenuation is observed in the case containing heavy inclusions. The increasing trend of these curves is due to the increased multiple scattering through the composite material as a result of an increase in the number of scatterers. One of the downsides of the Sabina-Willis model is that it cannot predict the resonant frequency shift due to variations in the inclusions concentration. Furthermore, the model is unable to predict other elastic resonant modes of the inclusions inside the matrix and hence gives rise to a single curve peak only.

\section{CONCLUSION}

This work analyzed the effective dynamic properties of composite materials containing randomly distributed inclusions for the incident $P$ and $S$ waves. From the methods, the Sabina-Willis model was selected for the analysis. The model performance was examined through a comparison with experimental results from the literature. Analyses were performed for different values of the physical and mechanical properties of inclusions relative to those of the matrix; more specifically, the cases with inclusions softer, stiffer, lighter, and heavier than the matrix were considered. The results indicated that, as the inclusion volume fraction increased, the normalized phase velocity increased in the composites with stiff or light inclusions but decreased in those with soft or heavy. Additionally, at a constant volume fraction in this model, the normalized attenuation in the composites containing soft and stiff inclusions was similar; whereas the attenuation of the composite with heavy inclusions was remarkably higher than that of the one containing soft inclusions. Furthermore, the model predicted the elastic resonant frequency in the composite containing stiff inclusions higher than that in the composite with soft inclusions. The rigid-body resonant frequency of the case with heavy inclusions was also observed to be lower than that of the case with light inclusions. It was shown that, in all of the cases, the effective dynamic properties of the medium were heavily affected by the propagated wave frequency and the inclusion volume fraction. However, such influence was insignificant at low volume fractions and low frequencies. Analysis of the effective dynamic properties for the two incidences of the P or S waves showed that, despite the different values obtained for the effective properties, their variation trends were closely similar.

Authors contribution: Conceptualization, M Rahimzadeh; Methodology, M Rahimzadeh; Investigation, M Rahimzadeh; Software, M Rahimzadeh; Validation, M Rahimzadeh; Writing - original draft, M Rahimzadeh; Writing - review \& editing, M Rahimzadeh.

Editor: Marco L. Bittencourt

\section{References}

Berryman, J. G. (1980). Long-wavelength propagation in composite elastic media II. Ellipsoidal inclusions. The Journal of the Acoustical Society of America, 68(6), 1820-1831.

Bringi, V. N., Varadan, V. V., \& Varadan, V. K. (1982). Coherent wave attenuation by a random distribution of particles. Radio Science, 17(5), 946-952.

Budiansky, B. (1965). On the elastic moduli of some heterogeneous materials. Journal of the Mechanics and Physics of Solids, 13(4), 223-227.

Christensen, R. M. (1990). A critical evaluation for a class of micro-mechanics models. Journal of the Mechanics and Physics of Solids, 38(3), 379-404.

Datta, S. K., Ledbetter, H. M., Shindo, Y., \& Shah, A. H. (1988). Phase velocity and attenuation of plane elastic waves in a particle-reinforced composite medium. Wave Motion, 10(2), 171-182.

Foldy, L. L. (1945). The multiple scattering of waves. I. General theory of isotropic scattering by randomly distributed scatterers. Physical review, 67(3-4), 107.

Hill, R. (1965). A self-consistent mechanics of composite materials. Journal of the Mechanics and Physics of Solids, 13(4), 213-222.

Kanaun, S. K., \& Levin, V. M. (2005). Propagation of shear elastic waves in composites with a random set of spherical inclusions (effective field approach). International Journal of Solids and Structures, 42(14), 3971-3997. 
Kanaun, S. K., \& Levin, V. M. (2007). Propagation of longitudinal elastic waves in composites with a random set of spherical inclusions (effective field approach). Archive of Applied Mechanics, 77(9), 627-651.

Kanaun, S. K., Levin, V. M., \& Sabina, F. J. (2004). Propagation of elastic waves in composites with random set of spherical inclusions (effective medium approach). Wave Motion, 40(1), 69-88.

Kim, J. Y., Ih, J. G., \& Lee, B. H. (1995). Dispersion of elastic waves in random particulate composites. The Journal of the Acoustical Society of America, 97(3), 1380-1388.

Kinra, V. K. (1985). Dispersive wave propagation in random particulate composites. In Recent Advances in Composites in the United States and Japan. ASTM International.

Kinra, V. K., \& Anand, A. (1982). Wave propagation in a random particulate composite at long and short wavelengths. International Journal of Solids and Structures, 18(5), 367-380.

Kinra, V. K., Day, N. A., Maslov, K., Henderson, B. K., \& Diderich, G. (1998). The transmission of a longitudinal wave through a layer of spherical inclusions with a random or periodic arrangement. Journal of the Mechanics and Physics of Solids, 46(1), 153-165.

Kinra, V. K., Ker, E., \& Datta, S. K. (1982). Influence of particle resonance on wave propagation in a random particulate composite. Mechanics research communications, 9(2), 109-114.

Kuster, G. T., \& Toksöz, M. N. (1974). Velocity and attenuation of seismic waves in two-phase media: Part I. Theoretical formulations. Geophysics, 39(5), 587-606.

Lax, M. (1951). Multiple scattering of waves. Reviews of Modern Physics, 23(4), 287.

Layman, C., Murthy, N. S., Yang, R. B., \& Wu, J. (2006). The interaction of ultrasound with particulate composites. The Journal of the Acoustical Society of America, 119(3), 1449-1456.

Li, R., Xia, H., Xu, Z., Ni, Q. Q., \& Fu, Y. (2017). U-DMA measurement and dynamic analysis of ultrasonic wave propagation in particulate composites. Composites Science and Technology, 151, 174-183.

Liu, D., \& Turner, J. A. (2008). Influence of spatial correlation function on attenuation of ultrasonic waves in two-phase materials. The Journal of the Acoustical Society of America, 123(5), 2570-2576.

Lloyd, P., \& Berry, M. V. (1967). Wave propagation through an assembly of spheres: IV. Relations between different multiple scattering theories. Proceedings of the Physical Society (1958-1967), 91(3), 678.

Luppé, F., Valier-Brasier, T., Conoir, J. M., \& Pareige, P. (2017). Coherent wave propagation in viscoelastic media with mode conversions and pair-correlated scatterers. Wave Motion, 72, 244-259.

Mal, A. K., \& Bose, S. K. (1974, November). Dynamic elastic moduli of a suspension of imperfectly bonded spheres. In Mathematical Proceedings of the Cambridge Philosophical Society (Vol. 76, No. 3, pp. 587-600). Cambridge University Press.

Markov, K. Z. (1999). On the correlation functions of two-phase random media and related problems. Proceedings of the Royal Society of London. Series A: Mathematical, Physical and Engineering Sciences, 455(1983), 1049-1066.

Markov, K. Z., \& Willis, J. R. (1998). On the two-point correlation function for dispersions of nonoverlapping spheres. Mathematical Models and Methods in Applied Sciences, 8(02), 359-377.

Mykhas'kiv, V. V., Khay, O. M., Zhang, C., \& Boström, A. (2010). Effective dynamic properties of 3D composite materials containing rigid penny-shaped inclusions. Waves in Random and Complex Media, 20(3), 491-510.

Mykhas'kiv, V., Kunets, Y., Matus, V., \& Khay, O. (2018). Elastic wave dispersion and attenuation caused by multiple types of disc-shaped inclusions. International Journal of Structural Integrity, 9(2), 219-232.

Rahimzadeh, M. (2013). Elastic wave propagation in nano-composites with random distribution of spherical inclusions. Latin American Journal of Solids and Structures, 10, 813-831.

Rahimzadeh, M., \& Daneshjoo, K. (2014). A comparative study on propagation of elastic waves in random particulate composites. Latin American Journal of Solids and Structures, 11(9), 1565-1590.

Sabina, F. J., \& Willis, J. R. (1988). A simple self-consistent analysis of wave propagation in particulate composites. Wave motion, 10(2), 127-142.

Sato, H., \& Shindo, Y. (2003). Multiple scattering of plane elastic waves in a particle-reinforced-composite medium with graded interfacial layers. Mechanics of Materials, 35(1-2), 83-106. 
Varadan, V. K., Ma, Y., \& Varadan, V. V. (1985). A multiple scattering theory for elastic wave propagation in discrete random media. The Journal of the Acoustical Society of America, 77(2), 375-385.

Waterman, P. C., \& Truell, R. (1961). Multiple scattering of waves. Journal of Mathematical Physics, 2(4), 512-537.

Wei, P. J., \& Huang, Z. P. (2004). Dynamic effective properties of the particle-reinforced composites with the viscoelastic interphase. International journal of solids and structures, 41(24-25), 6993-7007.

Willis, J. R. (1980). Polarization approach to the scattering of elastic waves-I. Scattering by a single inclusion. Journal of the Mechanics and Physics of Solids, 28(5-6), 287-305.

Yang, R. B. (2003). A dynamic generalized self-consistent model for wave propagation in particulate composites. J. Appl. Mech., 70(4), 575-582.

\section{Nomenclature}

$\kappa_{p, \text { eff }}$ Effective bulk modulus of the multi-phase medium in the case of $\mathrm{P}$ wave incidence.

$\mu_{p, \text { eff }}$ Effective shear modulus of the multi-phase medium in the case of $\mathrm{P}$ wave incidence.

$\rho_{p, e f f}$ Effective density of the multi-phase medium in the case of $\mathrm{P}$ wave incidence.

$\kappa_{s, e f f}$ Effective bulk modulus of the multi-phase medium in the case of $S$ wave incidence.

$\mu_{s, e f f}$ Effective shear modulus of the multi-phase medium in the case of $\mathrm{S}$ wave incidence.

$\rho_{s, e f f}$ Effective density of the multi-phase medium in the case of $\mathrm{S}$ wave incidence.

$\kappa_{2}$ Matrix bulk modulus.

$\mu_{2}$ Matrix shear modulus

$\rho_{2}$ Matrix density.

$\kappa_{1}$ Bulk modulus of inclusions.

$\mu_{1}$ Shear modulus of inclusions.

$\rho_{1}$ Density of inclusions.

$C_{p, e f f}$ Wave velocity in the multi-phase medium in the case of $\mathrm{P}$ wave incidence.

$C_{s, e f f}$ Wave velocity in the multi-phase medium in the case of $\mathrm{S}$ wave incidence.

$C_{p 2} \mathrm{P}$ wave velocity in the matrix.

$C_{s 2} \mathrm{~S}$ wave velocity in the matrix.

$k_{p, e f f} a$ Normalized wave number in the multi-phase medium in the case of $\mathrm{P}$ wave incidence.

$k_{s, e f f} a$ Normalized wave number in the multi-phase medium in the case of $\mathrm{S}$ wave incidence.

$k_{p 2} a$ Normalized wave number of the incident $\mathrm{P}$ wave.

$k_{s 2} a$ Normalized wave number of the incident $\mathrm{S}$ wave.

$c$ Volume fraction.

$a$ Inclusion's radius.

$r_{n+1}$ Matrix density.

$\mathbf{L}_{n+1}$ Matrix tensor of elastic moduli.

$\rho_{r}$ Density of each inclusion of type $r$.

$\mathbf{L}_{r}$ Tensor of elastic moduli of each inclusion of type $r$.

$\mathrm{x}^{\prime}$ Inclusion center.

$\Omega_{r}$ Shape of inclusion of type $r$. 
$n_{r}$ probability density function of inclusions of type $r$.

$c_{n+1}$ Matrix volume fraction.

$c_{r}$ Inclusion's volume fraction.

u Displacement field.

$\langle\mathbf{u}\rangle$ Mean displacement field.

$\sigma$ Stress tensor.

p Momentum density. 